\section{FRI0474 \\ FRACTURE RISK FOLLOWING BARIATRIC SURGERY: A SELF-CONTROLLED CASE SERIES AND RISK PREDICTION ALGORITHM}

Danielle E. Robinson ${ }^{1}$, lan Douglas ${ }^{2}$, Garry D. Tan ${ }^{3,4}$, Cyrus Cooper ${ }^{5,6}$, Antonella Delmestri ${ }^{1}$, Kassim Javaid Muhammad ${ }^{6}$, Andrew Judge ${ }^{1,7}$, Victoria Y. Strauss ${ }^{8}$, Daniel Prieto-Alhambra ${ }^{1} .{ }^{1}$ CSM, NDORMS, University of Oxford, oxford, United Kingdom; ${ }^{2}$ Faculty of Epidemiology and Population Health, London School of Hygiene and Tropical Medicine, London, United Kingdom; ${ }^{3}$ Oxford Centre for Diabetes, Endocrinology and Metabolism, Oxford University Hospitals NHS Foundation Trust, Oxford, United Kingdom; ${ }^{4}$ NIHR Oxford BRC, Oxford, United Kingdom; ${ }^{5}$ MRC Lifecourse Epidemiology Unit, University of Southampton, Oxford, United Kingdom; ${ }^{6}$ NDORMS, University of Oxford, Oxford, United Kingdom; ${ }^{7}$ Translational Health Sciences, Bristol Medical School, University of Bristol, Bristol, United Kingdom; ${ }^{8}$ CSM, NDORMS, University of Oxford, Oxford, United Kingdom

Background: Bariatric surgery is increasingly common due to the obesity epidemic. However, there is controversial evidence about the association between bariatric surgery and fracture risk. This may be due to the fundamental differences between patients undergoing bariatric surgery with patients who do not, raising the question: within patients who fracture, are they are at increased risk of fracture after surgery? And if so, who is at risk of fracture?

Objectives: 1. To investigate the association between bariatric surgery and risk of three fracture locations using a within person study design comparing a) the 5 year incidence post surgery to the 5 year pre surgery, b) splitting the 5 year post surgery risk into two windows $0-2$ and $2.01-5$ years. 2. To predict who is at risk of fracture following bariatric surgery.

Methods: A self-controlled case series analysis (SCCS) and Stepwise logistic regression (LR) were conducted. Patients undergoing bariatric surgery were identified in the clinical practice research datalink (CPRD) GOLD dataset and linked to hospital episode statistics (HES) data. Primary outcome was any fracture (any skeletal sites except skull and digits). Secondary outcomes were major (hip, spine, forearm and humerus) and peripheral fractures (forearm and lower leg). For the SCCS, Poisson models in those who fractured were fit to calculate Incidence Rate Ratios (IRR) for the aforementioned time windows. Potential predictors were selected with an exit $p$-value of 0.157 and their predictive performance was reported using a C-statistic.

Results: Of 5,492 patients undergoing bariatric surgery, 252 patients had 272 any fractures, 75 (80) major fractures and 126 (135) peripheral fractures. Average BMI was 43.9. Major fracture risk increased nearly threefold following surgery: IRR $(95 \% \mathrm{Cl}) 2.70$ (1.31, 5.57). Conversely, the incidence of any and peripheral fractures did not change: IRRs 1.17 $(0.86,1.60)$ and $0.92(0.60,1.42)$ respectively. Any and major fracture risk increased in the $3^{\text {rd }}$ to $5^{\text {th }}$ year post-surgery: IRRs of $1.73(1.08$, 2.77), $4.98(1.94,12.78)$ respectively. 7 variables were identified which may predict major fracture with a high c-statistics of $0.77(0.71,0.88)$.

Conclusion: Few patients had fractures (252/5492). The incidence of major osteoporotic fracture is increased after bariatric surgery by nearly 3 fold, to then increase to 5 fold in the 2-5 year window. Multiple factors were identified for patients at risk of major fracture post-operatively. External validation is needed. Further research is needed on post-bariatric surgery care to minimise fracture risk.

Acknowledgement: This project was funded by the NIHR RfPB (project number PB-PG-1215-20017) and supported by the NIHR Oxford BRC. The views expressed are those of the author(s).

Disclosure of Interests: Danielle E Robinson: None declared, lan Douglas: None declared, Garry D Tan: None declared, Cyrus Cooper Consultant for: Personal fees from Alliance for Better Bone Health, Amgen, Eli Lilly, GSK, Medtronic, Merck, Novartis, Pfizer, Roche, Servier, Takeda and UCB., Antonella Delmestri: None declared, Kassim Javaid Muhammad Shareholder of: Zebra Medical Vision, Grant/research support from: Amgen, Kyowa Kirin Hakin

Consultant for: Amgen, Internis, Consilient Health,Mereo Biopharma, Kyowa Kirin Hakin, UCB, Speakers bureau: Amgen, Lilly UK, Internis, UCB, Andrew Judge Grant/research support from: Consortium research grants from Roche, Consultant for: Received consultancy fees, lecture fees and honoraria from Servier, UK Renal Registry, Oxford Craniofacial Unit, IDIAP Jordi Gol and Freshfields Bruckhaus. Member of Deringer Data Safety and Monitoring Board for Anthera Pharmaceuticals, Inc. Consultancy for Freshfields Bruckhaus Deringer, Victoria Y Strauss: None declared, Daniel Prieto-Alhambra Grant/research support from: Grants from Amgen, UCB Biopharma and Servier outside the submitted work, Consultant for: UCB Biopharma, Speakers bureau: Amgen

DOI: 10.1136/annrheumdis-2019-eular.2437
FRI0475

TITLE: LUMBAR SPINE AND PROXIMAL FEMUR OSTEOPOROSIS DIAGNOSIS: REMS TECHNOLOGY IN A MULTICENTER CLINICAL STUDY

Giovanni Adami ${ }^{1}$, Giovanni Arioli ${ }^{2}$, Gerolamo Bianchi ${ }^{3}$, Maria Luisa Brandi ${ }^{4}$, Carla Caffarelli ${ }^{5}$, Loredana Cavalli ${ }^{4}$, Marco Matucci-Cerinic ${ }^{6}$, Luisella Cianferotti ${ }^{4}$, Francesco Conversano ${ }^{7}$, Marco DI Paola ${ }^{7}$, Davide Gatti ${ }^{1}$, Giuseppe Girasole ${ }^{3}$, Andrea Giusti ${ }^{3}$, Stefano Gonnelli ${ }^{5}$, Monica Manfedini ${ }^{2}$, Maurizio Muratore ${ }^{8}$, Ranuccio Nuti ${ }^{9}$, Paola Pisani ${ }^{7}$, Laura Quarta ${ }^{10}$, Ombretta Viapiana ${ }^{11}$

${ }^{1}$ Rheumatology Unit, Department of Medicine, University of Verona, Verona, Italy; ${ }^{2}$ Department of Neurosciences and Rehabilitation, "Carlo Poma" Hospital, ASSTMantova, Mantova, Italy; ${ }^{3}$ SC Rheumatology, ASL 3 Genovese, Genoa, Genoa, Italy; ${ }^{4}$ Department of Surgery and Translational Medicine, University of Florence, Metabolic Bone Diseases Unit, University Hospital of Florence, Florence, Italy; ${ }^{5}$ Department of Medicine, Surgery and Neurosciences, University of Siena, Siena, Italy; ${ }^{6}$ Department of Experimental and Clinical Medicine, University of Florence and SOD Rheumatology AOUC, Florence, Florence, Italy, ${ }^{7}$ National Research Council, Institute of Clinical Physiology, Lecce, Lecce, Italy, ${ }^{8}$ O.U. of Rheumatology, "Galateo" Hospital, San Cesario di Lecce, ASL-LE, Lecce, Lecca, Italy; ${ }^{9}$ Department of Medicine, Surgery and Neurosciences, University of Siena, Siena, Italy, ${ }^{10}$ O.U. of Rheumatology, "Galateo" Hospital, San Cesario di Lecce, ASL-LE, Lecce, Lecce, Italy, ${ }^{11}$ Rheumatology Unit, Department of Medicine, University of Verona, Verina, Italy

Background: To date numerous quantitative methods to diagnose osteoporosis at peripheral sites have been developed. REMS (Radiofrequency Echographic Multi Spectrometry) is a non-ionizing instrument used to quantitatively evaluate bone mineral density at lumbar spine and femoral neck sites [1].

Objectives: To assess the accuracy of REMS in osteoporosis diagnosis at axial sites (lumbar spine and hip) compared with the gold standard, Dual-energy X-ray absorptiometry (DXA).

Methods: We enrolled patients aged from 50 to 75 years and perfomed a total of 1,699 and 1,794 lumbar spine and proximal femur scans with REMS and DXA. A quality checks on DXA and REMS acquired data were performed through direct examination of scans by experienced operators. We investigated the agreement between the two techniques calcu lating: Pearson's correlation coefficient $(r)$, Standard Error of the Estimate (SEE) and efficiency (sensitivity and specificity) in identifying osteoporotic individuals (i.e. T-score $\leq-2.5$ )

Results: We found a good linear correlation between REMS and DXA BMD estimation, Pearson coefficient $r=0.938$ for lumbar spine and $r=0.933$ for proximal femur ( $p<0.001$ for both). SEE values were $5.2 \%$ and $5.8 \%$ for lumbar spine and femoral site, respectively. In addition, REMS demonstrated a high sensitivity $(92.9 \%$ and $92.3 \%$ for spine and femur) and specificity $(93.5 \%$ for spine and $92.6 \%$ for femur) for the discrimination of osteoporotic patient. Moreover, considering together all the diagnostic classification categories (healthy, osteopenia and osteoporosis), the diagnostic agreement between REMS and DXA was $90.2 \%$ and $89.3 \%$ for lumbar spine and proximal femur sites, respectively.

Conclusion: REMS exhibited a high sensitivity and specificity in identifying osteoporotic patients at lumbar spine or proximal femur sites compared with gold-standard DXA. REMS accurately diagnosed osteoporotic patients in clinical routine, at both lumbar spine and proximal femur sites, without using ionizing radiation.

\section{REFERENCES:}

[1] Di Paola M, Gatti D, Viapiana O, et al. Osteoporos Int, 2018

Disclosure of Interests: Giovanni Adami: None declared, Giovanni Arioli: None declared, Gerolamo Bianchi: None declared, Maria Luisa Brandi: None declared, Carla Caffarelli: None declared, Loredana Cavalli: None declared, Marco Matucci-Cerinic Grant/research support from: Actelion, MSD, Pfizer, BMS, Chemomab, Sanipedia, Speakers bureau: Actelion, BMS; MSD, Janssen, Luisella Cianferotti Speakers bureau: Abiogen Pharma, Bruno Farmaceutici, Shire, Francesco Conversano Shareholder of: Francesco Conversano own stocks of Echolight Spa, Marco Di Paola: None declared, Davide Gatti Speakers bureau: Abiogen, Amgen, JanssenCilag, Mundipharma, Pfeizer, Giuseppe Girasole: None declared, Andrea Giusti: None declared, Stefano Gonnelli: None declared, Monica Manfedini: None declared, Maurizio Muratore: None declared, Ranuccio Nuti: None declared, Paola Pisani: None declared, Laura Quarta: None declared, Ombretta Viapiana Speakers bureau: Novartis, Abbvie, Eli-Lilly, Sanofi Genzyme

DOI: 10.1136/annrheumdis-2019-eular.6256 